\title{
Is Longevity Acceleration Sustainable? An Entropy-Based Trial of the Population of Spain vs. Japan
}

\author{
Amancio Betzuen Zalbidegoitia ${ }^{1}$ and Amaia Jone Betzuen Álvarez ${ }^{2, * \mathbb{D}}$ \\ 1 Department of Economics and Finance I, Economics and Business Faculty, Av/Lehendakari Agirre 83, \\ Campus of Vizcaya, University of the Basque Country, 48015 Leioa, Spain; amancio.betzuen@ehu.eus \\ 2 Department of Economics and Finance II, Economics and Business Faculty, Av/Lehendakari Agirre 83, \\ Campus of Vizcaya, University of the Basque Country, 48015 Leioa, Spain \\ * Correspondence: amaiajone.betzuen@ehu.eus or amaiabetzuen@outlook.com
}

check for

updates

Citation: Betzuen Zalbidegoitia, A.; Betzuen Álvarez, A.J. Is Longevity Acceleration Sustainable? An Entropy-Based Trial of the Population of Spain vs. Japan. Mathematics 2021, 9,1810. https://doi.org/10.3390/ math9151810

Academic Editors: Juan

Jose García-Machado and

Octavian Dospinescu

Received: 27 May 2021

Accepted: 28 July 2021

Published: 30 July 2021

Publisher's Note: MDPI stays neutral with regard to jurisdictional claims in published maps and institutional affiliations.

Copyright: (C) 2021 by the authors Licensee MDPI, Basel, Switzerland. This article is an open access article distributed under the terms and conditions of the Creative Commons Attribution (CC BY) license (https:/ / creativecommons.org/licenses/by/ $4.0 /)$.

\begin{abstract}
Longevity risk is a major concern for governments around the world as they have to address social benefits, whether in the form of pensions, healthcare, or caring for dependents and providing long-term care, and so forth, which directly impact countries' budgets. This paper uses a single entropy index to measure this type of risk. This methodology is clearly different from the one traditionally used in the literature, which is nearly entirely based on measuring the evolution of mathematical life expectancy. The authors used the longest-living populations in the world, Japan and Spain, to create a database in order to analyse the virtue of the indicator. The aim was to establish whether the longevity of those populations is accelerating or decelerating, compared by sex, and whether that occurs at the same intensity at different stages of a person's life in each case. If the indicator showed differences in intensity, it would be a benchmark for the insurance and financial industry, providing it with information to market different products.
\end{abstract}

Keywords: longevity; mortality risk; entropy; measurement; sustainability; Japan; Spain

\section{Introduction}

During recent decades studying and researching longevity risk, we have found periods when the survival of people progressed slowly and periods with spectacular advances in longevity, as has been the case in developed countries in recent decades. This longevity acceleration has created huge problems in the majority of countries, as well as for insurers, the Social Security system, pension funds, and so forth.

Many authors have published papers on this subject, and the majority have used indicators such as the mathematical life expectancy. Those results have been reflected on a survival curve. The curve shows that the further to the right it evolves, and depending on the person's age, the longer the longevity of the population. However, this attractive way of presenting the obtained results is not the most appropriate from a quantitative point of view.

Our index is different and possibly more abstract and objective. On the one hand, this allows us to provide the longevity evolution results using a different methodology and, on the other hand, to compare the longevity evolution between two different countries with such long lifespans as Japan and Spain.

Even though this index, as such, is used objectively, our approach will closely connect its size to the real risk situation of a population. Thus, the results obtained will allow us to affirm whether the level of longevity risk of a country is accelerating or decelerating, and, furthermore, if that acceleration or deceleration is uniform for the whole life timeline of an individual or if it differs between certain age brackets. This is a clear difference with respect to the life expectancy indicator.

The methodology to show the advance of longevity applied in this paper has not been used in the field of demographics but has in the information field. In general, the 
entropy index applied in this paper is a method to measure divergences between targets, capital flows, and information differences. The life expectancy indicator does not measure such divergences.

In the literature, entropy has been used as a method to measure information distribution or analyse differences or heterogeneity when measuring the health difference between two territories or two populations in which the differences regarding income level are more than significant. It has also been used to assess the evolution of different monetary flows, different environmental conditions, nutrition levels and so forth. Therefore, we contribute the use of a different indicator to the mathematical life expectancy, which has the ability to show the divergence or not between two distributions. To the best of our knowledge, the way in which it has been applied has not been previously used to measure the difference in the survival improvement between two different countries. Furthermore, we contribute the possibility of determining whether the improvement to longevity is distributed in a more or less uniform way or whether it shows different intensities depending on the age range considered. Furthermore, we want to make it clear that the presentation of this evidence has the appeal that it can be used by the insurance industry, among others, given that the results obtained directly influence the price of the insurance product marketed.

\section{Literature Review}

Given the explosive impact of the evolution of longevity worldwide, there are many papers that have sought to study and quantify its impact as far as possible [1,2]. There are studies in the literature focused exclusively on the Japanese population, given its great advances in survival [3]. Those studies focus on general mortality, where the future population survival trend is estimated, taking total gross data into consideration and without distinguishing by sex.

We have also found longevity risk studies focused on Netherlands [4] and Nordic countries, Norway or Sweden [5]. The survival studies in the last of those countries are highly productive as extensive historical information is available on the mortality of the general population.

The majority of papers use the Lee-Carter model (readers interested in the characteristics of that model will find further information in the References section [6-10]) to perform future mortality estimates. The validity of the model is proven and accepted in the literature and is widely used to perform survival forecasts based on historical data. In that regard, we should note that there are other models in the literature for the same purpose, i.e., studying the improvement to mortality rates, albeit along different paths [11-14].

We have also used this methodology in different papers presented at conferences and in publications. However, we have always applied the aforementioned model to estimate different parameters, which leads to the possibility of determining the mathematical life expectancy. Yet, there are very few papers focused on the study of the acceleration or deceleration of improvements in mortality. The use of entropy is progress on the preceding studies given that what is determined is not whether survival advances, which is obvious and is contrasted, but rather whether its advance gains in intensity, remains steady, or if it decreases. The prognosis changes completely in any of those situations.

The methodology used in this paper to determine the advance in longevity does not come from the field of demographics. Its origins lie in the information field, where it has been widely used as a method to measure divergences between targets, flows of quantities, types of information, etc. The analysis of differences or of heterogeneities serves as information when measuring the difference regarding the income level in a population considered as different monetary flows, differences in nutrition levels, differences in educational levels, etc. Consequently, when turning to the demographic field, we need information specific to the evolution of different flows that can be compared for a single reference period. This methodology, unlike the classical one, allows to measure the speed of the improvement to survival and even its acceleration, should that be the case, be 
researched. Furthermore, whether that improvement is uniform, whether it is evolving in the same direction, or whether it differs by age groups can be established.

The first studies that applied entropy used information indicators [15,16], but its indexes were based on data related to information in general. The first studies into the use of entropy to measure the differences between populations focused on different criteria, including race and sex.

More recently, studies where the entropy concept is linked to the study of the rectangularization of human survival $[13,17,18]$ have been presented. They argue that improvements in mortality have been mainly due to advances in older ages. Those aspects differ from our findings. New contributions have recently been made to the study of the progress of rectangularization in the field of biometrics $[19,20]$.

Once the problem in question has been put into context, the existing literature reviewed and the objective specified, this research paper is structured as follows: the next point describes the methodology used to achieve the objectives, along with the database established to be used; the results obtained are shown in the fifth point and the sixth, presents the most important evidence and conclusions. The paper ends with references we have considered appropriate to underpin and channel our paper.

\section{Research Objectives}

Starting from the above premises, we seek to use the entropy indicator to measure the improvement in mortality as an alternative to life expectancy, survival curve, or rectangularization.

Furthermore, we seek to ascertain whether this indicator is able to capture the divergence between two distributions of improved longevity for the chosen populations in the study, Japanese and Spanish, as they represent two countries with current low mortality and proven longevity.

\section{Materials and Methods}

As already mentioned in the above section, this paper uses a new method to measure survival entropy measurement for two populations, which are Spain and Japan. They were mainly chosen for their spectacular progress in improving survival compared in global terms in the last thirty years.

We carefully selected the information sources to conduct our study. Given that we were going to apply the tool to measure the longevity risk, we decided to proceed in two stages: the first involved adequately setting up the baseline database, and the second, transforming the entropy formula to be used in our calculations.

We obtained the values for the entropy indexes using the gross values of the death rates, which are publicly and freely available from the Human Mortality Database (hereinafter HMD) [21]. Their origin is due to the first-rate work of the Department of Demography at the University of California, Berkeley, and the Max Planck Institute for Demographic Research in Rostock. That database provides the recent gross data for both countries and for both sexes. Using these data sources guarantees that information is obtained from the same source and under the same conditions for both countries. Thus, we believe that it guarantees that the entropy measurement provides the differences in improvement, if they exist, between countries, for the same sex, solely due to acceleration or deceleration.

However, given that the data range does not match the source of origin, we have limited that range to the same age brackets and calendar time. On the other hand, the gross data published by the HMD for the longest-living ages do not provide results that can be considered adequate for the years that they represent. We noted that same gross rates are non-existent in real life for some ages or present a value over one, which is unacceptable from the statistical point of view. Therefore, we proceeded to eliminate the data over the age of 105 years old and perform a smoothing, as will be explained later. This whole process to analyse gross data from the HMD, and prior to their use, had been performed in some of our previous research work. It required a preliminary in-depth study, such as establishing 
a longevity index that could be used by the different insurance and financial entities in order to guarantee the cover of their private pension provisions, life insurance, retirement annuities, inter alia, for its clients. Such an index is known as a longevity index [7-9].

Therefore, the preparation of our database complies with the guidelines defined in the Technical Document known as Life Metrics [22], which establishes precise criteria for a study forecasting the survival of a cohort of people to be considered valid. In our opinion, very few studies take this publication into account. Suffice to say that its compliance requires a painstaking debugging of the database prior to applying a survival model.

Following the guidelines of the aforementioned document and using the Lee-Carter model [10], we have managed to set up a database with adequate regularity in the evolution of improvement to mortality rates during the calendar years.

In order to perform the comparison between countries in the most similar conditions as possible, we chose the calendar year period from 1960 to 2018, when we observed the most notable improvement in the second part of the past century. As regards the age range, we chose the total lifecycle span of a person, from age zero until the final age that a member of the collective can reach, which we denote by the letter $w$. However, we pay special attention to the age ranges under and over 65 years old in order for our finding to be useful for the insurance industry.

The baseline database has been smoothed using the following model that, essentially, consists of applying an equation as follows:

$$
f(x, t)=m_{x, t}=\exp \left(a_{x}+b_{x} * k_{t}+\varepsilon_{x, t}\right)
$$

where (the numerical values of those parameters are not present in this work in order to simplify the presentation but are available on request from the lead researcher. See [8] for some results for a study on the longevity of a population):

$m_{x, t}:$ represents the central death rates obtained as the quotient between the number of deaths for each individual age and for each calendar year and the number of years of exposure to risk by the individuals and years in question.

$a_{x}$ : represents the average value, in logarithmic terms, of the way mortality behaves according to age. This variable does not depend on the calendar time.

$b_{x}$ : represents the speed of the improvement according to age. This variable does not depend on the calendar time.

$k_{t}$ : represents the evolution of the improvement according to the calendar year.

$\varepsilon_{x, t}$ : represents the error variable.

We used an R package for stochastic mortality modeling called StMoMo [23]. That statistical software has provided us with the values of the parameters shown graphically below.

For the case of the Spanish population, the values of the $a_{x}$ parameter according to the age of the individual are those shown in Figure 1:

The graph on the left is for the male population and that on the right for the female population. The value for the $a_{x}$ parameter can be seen to comply with two fundamental requirements for all ages. On the one hand, it is within the upper (red line) and lower (gray line) limits of the values given by the gross mortality rate logarithm. The upper limit is for the first calendar year of our database (1960), and the lower limit is for the last calendar year (2018). On the other hand, its evolution is much more regular, complying with one of the objectives set by us when establishing the baseline database to determine the entropy index. 


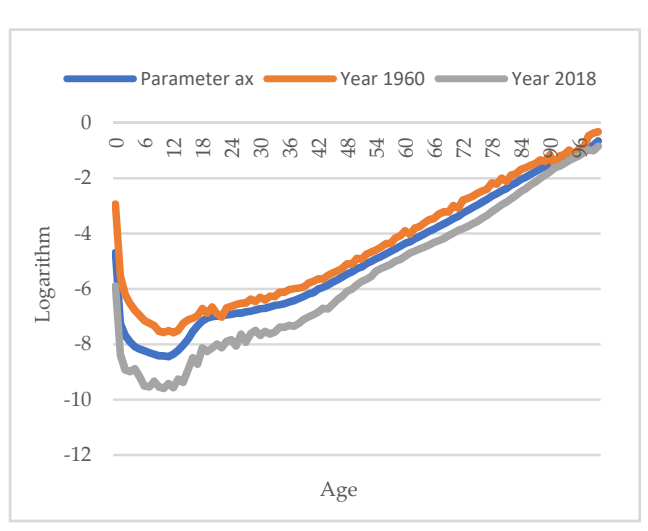

(a)

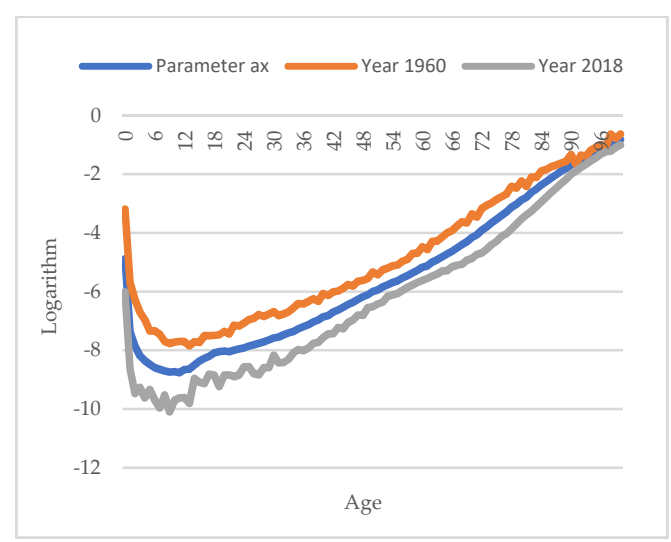

(b)

Figure 1. (a) Values of the $a_{x}$ parameter according to the age for the Spanish male population and gross values of the mortality rates of the extreme calendar years; (b) values of the $a_{x}$ parameter according to the age of the Spanish female population and gross values of the mortality rates of the extreme calendar years. Source: authors.

For the case of the Japanese population, the values of the $a_{x}$ parameter according to the age of the individual are shown in Figure 2:

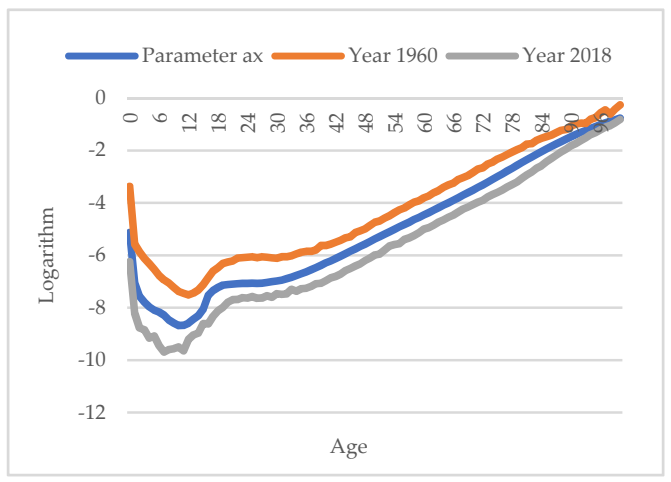

(a)

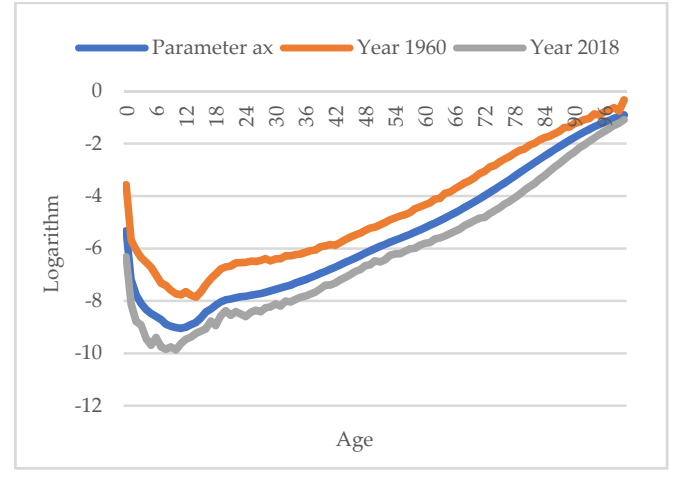

(b)

Figure 2. (a) Values of the $a_{x}$ parameter according to the age for the Japanese male population and gross values of the mortality rates of the extreme calendar years; (b) values of the $a_{x}$ parameter according to the age for the Japanese female population and gross values of the mortality rates of the extreme calendar years. Source: authors.

In a similar way to the case of the Spanish population, the parameter for the Japanese population behaves within the established margins and also regularly.

Figure 3 below shows the behavior of the age parameter $b_{x}$ and of the time parameter $k_{t}$. In the interest of simplification, we only show the result obtained for the Spanish male population where the regular behavior of parameter $b_{x}$, obtained by applying the Lee-Carter model [10]. Please note that we obtained similar behaviors of that parameter for the other populations studied, i.e., the Japanese and Spanish female population and the Japanese male population. 


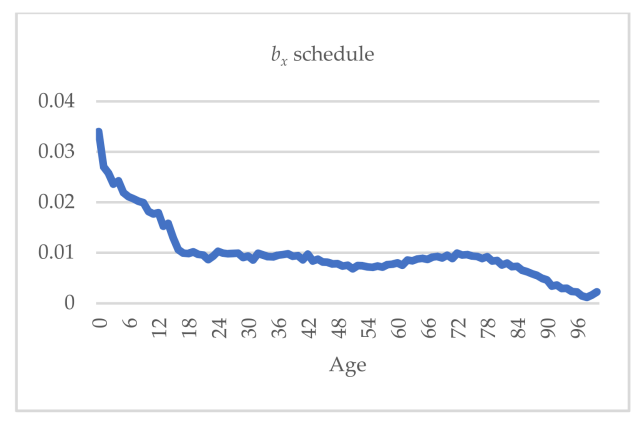

(a)

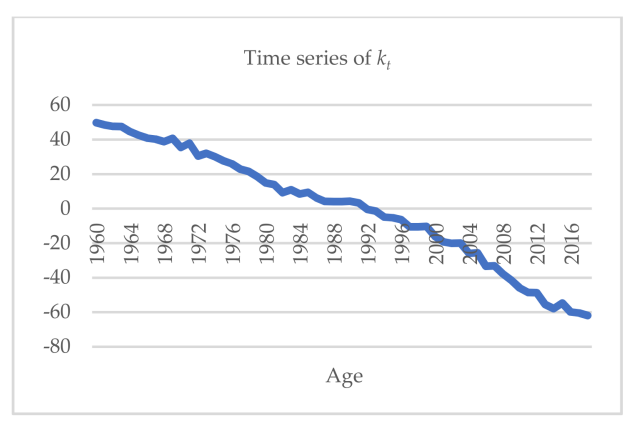

(b)

Figure 3. (a) Age parameter $b_{x}$ for the Spanish population; (b) time parameter $k_{t}$ for the Spanish population. Source: authors.

The time parameter $k_{t}$ likewise shows a regular evolution with a clear trend to improving survival, as can be seen by its downward line. This trend is similar to the other cohorts studied.

We have thus smoothed the gross baseline data, which were obtained directly from the HMD database. The data thus constituted form the baseline database and are used to establish the entropy measurement.

The methodology that we want to explore is based on the entropy measurement provided by the following expression:

$$
H_{x}=-\frac{\int_{x}^{w}[\ln l(t)] * l(t) d t}{\int_{x}^{w} l(t) d t} 0 \leq x \leq w
$$

In the formula defined by Keyfitz [15], $H_{x}$ represents the entropy at specific age $x$. On the other hand, $l(t)$ represents the survival function and $w$ the final age of the mortality table.

The problem we come across is that, in practice, the baseline values to be used for the calculation are provided discretely. This is the case in the publications provided worldwide by the HMD. However, there are biometric relations in the field of demographics in order to use continuous values. For example, by adjusting a biometric function that better represents the evolution of a survival curve created using the data published by HMD. We have opted to take the data directly from HMD, which we smooth using the Lee-Carter model [10], and transform the previous formula given from continuous to discrete form:

$$
H_{x}=-\frac{\Sigma_{x}^{w}[\ln l x] l x}{\Sigma_{x}^{w} l x} 0 \leq x \leq w
$$

On the other hand, the baseline data correspond to the gross central mortality rates and must be transformed into gross annual rates and, therefore, into the survival function $l(x)$. All these relations can be found in any actuarial biometric manual.

It is obvious that, if the function $l(x)$ represents the survival curve in the continuous field, the area representing the lower part of the curve is higher than the values that the summation operator provides, but those values are reduced to negligible amounts when the intervals are monthly, instead of annual periods, which is how they are presented in the HMD publications. This partition does not present any difficulty, as it can be performed using any of the methods in the actuarial biometric manuals. The minus sign in the formula is necessary to obtain positive values for the entropy measurement.

Other approximate formulas can be used to measure entropy and can be deduced from the contributions of different authors $[17,24,25]$, based on the definition of entropy in Keyfitz's publication [15]. Another interesting contribution [12,25], as it incorporates the valuation interest rate, along with the instantaneous rate of mortality, can also be consulted. Logically, the size of the entropy measurements not only varies, in this case, according 
to the improvement to mortality but also to the discount interest rate applicable on the insurance product market or that authorized by the General Insurance Directorate.

\section{Results}

Proceeding as described in Section 4 on the methodology used in the study and applying the above Formula (3) to the male populations of Japan and Spain, we obtain the results shown in the following Table 1 :

Table 1. Keyfitz entropy measurement [15] for the male population for each specific age.

\begin{tabular}{ccc}
\hline Age & Japan & Spain \\
\hline 5 & 0.126 & 0.130 \\
10 & 0.135 & 0.138 \\
15 & 0.145 & 0.149 \\
20 & 0.156 & 0.160 \\
25 & 0.169 & 0.174 \\
30 & 0.185 & 0.190 \\
35 & 0.203 & 0.210 \\
40 & 0.226 & 0.234 \\
45 & 0.253 & 0.263 \\
50 & 0.288 & 0.301 \\
55 & 0.332 & 0.349 \\
60 & 0.391 & 0.412 \\
65 & 0.469 & 0.496 \\
70 & 0.578 & 0.610 \\
75 & 0.733 & 0.773 \\
80 & 0.967 & 1.023 \\
85 & 1.352 & 1.432 \\
90 & 2.012 & 2.135 \\
\hline
\end{tabular}

Source: authors' calculation.

The entropy measurement can be seen to increase with age, slowly at first and at greater intensity from middle age. The difference between the two populations is not significant, except from middle age such as at 50 years old.

As has already been mentioned, the entropy measurement allows us to determine the improvement percentage to mathematical life expectancy. When calculating entropy at age zero, we note how its value has gradually fallen with the passing of time, meaning life expectancy at the origin of life has improved. This can be interpreted as that the number of elderly survivals has been increasing, and, consequently, so has the longevity of the population.

Figure 4 compares both countries, Japan and Spain, to show the advance in longevity.

Even though the entropy measurement for young ages does not provide notable information on the improvement to mortality among the countries being studied, that measurement shows greater acceleration in the Japanese population for older ages, with the distribution of the acceleration being regular in both countries. 


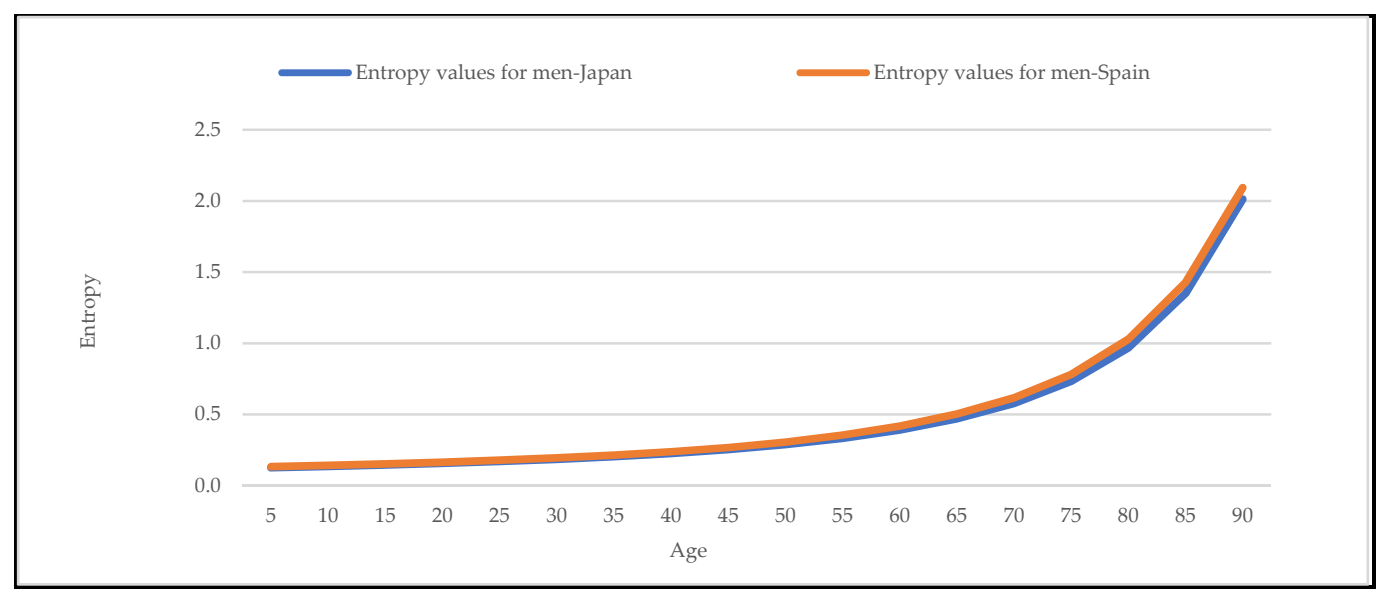

Figure 4. Japan vs. Spain entropy measurements for the male population according to the age of the individual. Source: authors.

If we analyse the results for women, we obtain Table 2:

Table 2. Keyfitz entropy measurement [15] for the female population for each specific age.

\begin{tabular}{ccc}
\hline Age & Japan & Spain \\
\hline 5 & 0.102 & 0.101 \\
10 & 0.108 & 0.108 \\
15 & 0.115 & 0.115 \\
20 & 0.123 & 0.123 \\
25 & 0.133 & 0.133 \\
30 & 0.144 & 0.144 \\
35 & 0.157 & 0.158 \\
40 & 0.172 & 0.174 \\
45 & 0.191 & 0.193 \\
50 & 0.214 & 0.217 \\
55 & 0.242 & 0.248 \\
60 & 0.278 & 0.287 \\
65 & 0.325 & 0.339 \\
70 & 0.390 & 0.411 \\
75 & 0.482 & 0.517 \\
80 & 0.624 & 0.686 \\
85 & 0.857 & 0.977 \\
90 & 1.271 & 1.514 \\
\hline
\end{tabular}

Source: authors' calculation.

The similarity of the entropy measurement between those two populations for the under-50 s is surprising. In the same way, as for the male collective, entropy increases with the specific age of the individual, and this accelerates to a greater extent among the Japanese female population, with the distribution of the acceleration being regular in both countries.

We should note that, in this case, unlike the male population, the entropy measurement shows a greater gap between both countries for older ages, but the acceleration is smaller than in the case of men.

Figure 5 on entropy with respect to age, compared by countries, provides a benchmark for the difference in the number of survivals, particularly when older. Given that the entropy values are lower in the Japanese population, we can interpret it as the relative difference in the survival probability favorable to the Japanese population and, therefore, to life expectancy. 


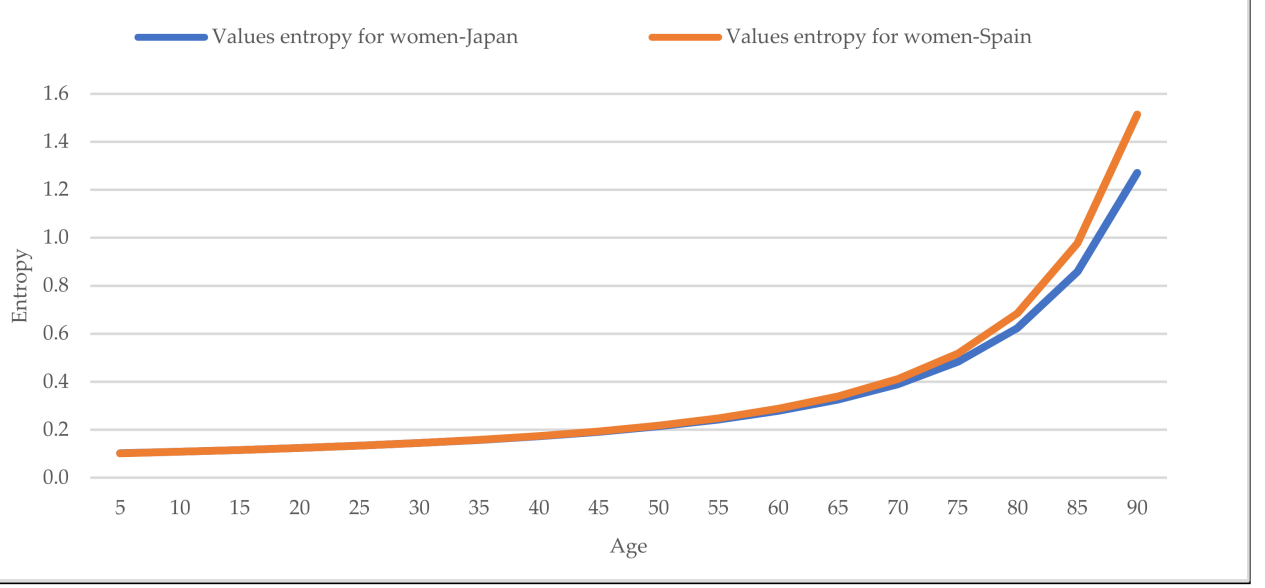

Figure 5. Japan vs. Spain entropy measurements for the female population according to the age of the individual. Source: authors.

When this curve is analyzed in greater detail, it can be seen that, roughly speaking, the evolution of this curve has a certain similarity with the reverse evolution of the mathematical life expectancy, in general, and for certain broad age groups. Choosing an adjustment function frequently used in statistical regressions suffices to corroborate it.

This behavior of entropy had caused some researchers to claim that there is a clear reverse relationship between entropy and mathematical life expectancy [26]. We chose the values set out in Table 2 to check this claim. We contrasted them with the values of the mathematical life expectancy emerging from the database that we constructed to apply entropy.

The adjustment formula that fits the aforementioned conditions, i.e., the entropy curve, is as follows:

$$
H(x)=a+b\left(\frac{1}{E(x)}\right)+c\left(\frac{1}{E(x)}\right)^{2}
$$

The following values of the parameters in Table 3 are obtained by applying ordinary least squares:

Table 3. Values of the parameters of Formula (4).

\begin{tabular}{ccccc}
\hline & $\boldsymbol{a}$ & $\boldsymbol{b}$ & $\boldsymbol{c}$ & $\boldsymbol{R}^{\mathbf{2}}$ \\
\hline Spain & 0.024133 & 8.376782 & 39.59566 & 0.99985 \\
Japan & 0.028789 & 7.829319 & 37.86558 & 0.99972 \\
\hline
\end{tabular}

Source: authors' calculation.

It can be seen that the adjustment measured by the $R^{2}$ presents an excellent result. Therefore, the relationship between the entropy and the reverse of the mathematical life expectancy is justified.

Additionally, we present Figure 6 below, showing the adjustment performed based on the results presented in Table 3: 


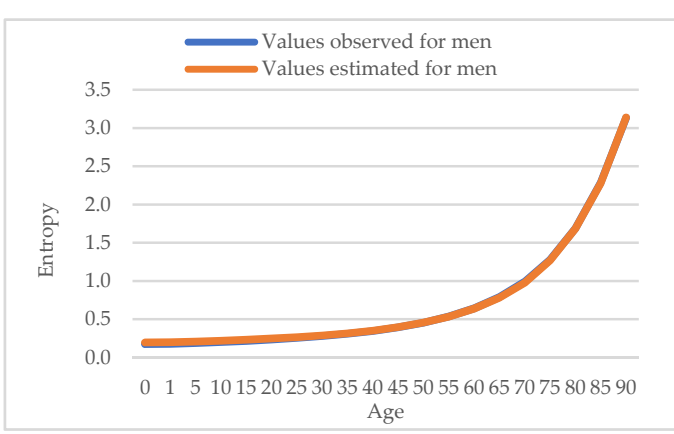

(a)

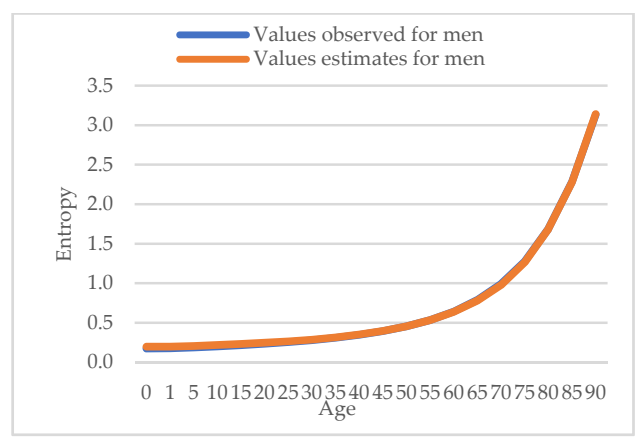

(b)

Figure 6. (a) Adjustment for the case of Spain; (b) adjustment for the case of Japan. Source: authors.

It can be seen at a glance that the two curves practically overlap in both countries. However, a more precise analysis reveals that they differ by a very small amount in the early ages.

We checked that the evolution of the entropy measurement according to the ages of the individual does not present ambiguities. Its evolution is regular and clearly shows some differences between cohorts more clearly than the survival curve. We then embarked on establishing whether this regular behavior likewise presents over the calendar years.

We chose three sufficiently representative ages, 40, 65, and 90 years old, in order to simplify the reasoning. The first is the age on entering maturity and the usual age for contracting general insurance such as life and traffic. The second, the age, which marks the start of many status changes, such as the end of the working life and moving from receiving a salary to collecting social benefits and the usual age for contracting retirement income. The last represents the fourth age population when reverse mortgage, dependency, long-term care insurance, among others, are taken out.

\subsection{Detailed Analysis of the Results Obtained for the Male Populations}

We provide the following Figures 7-9 for the comparative analysis for the male case:

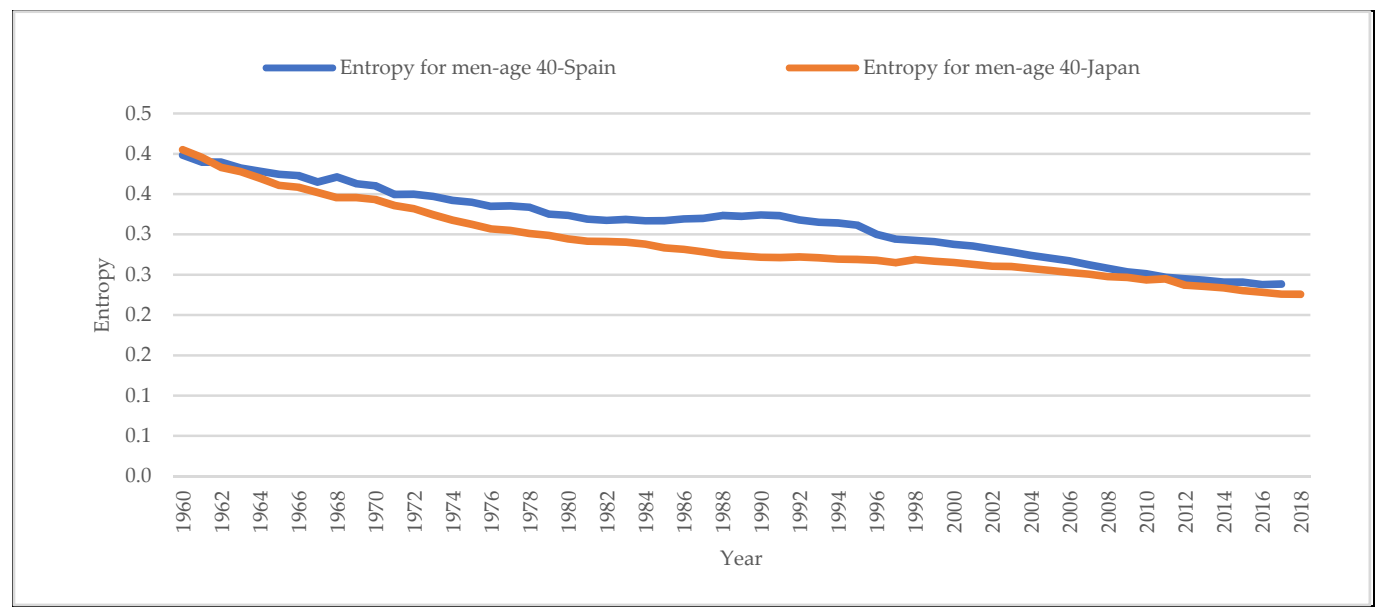

Figure 7. Entropy evolution for the male population at 40 over calendar years. Source: authors. 


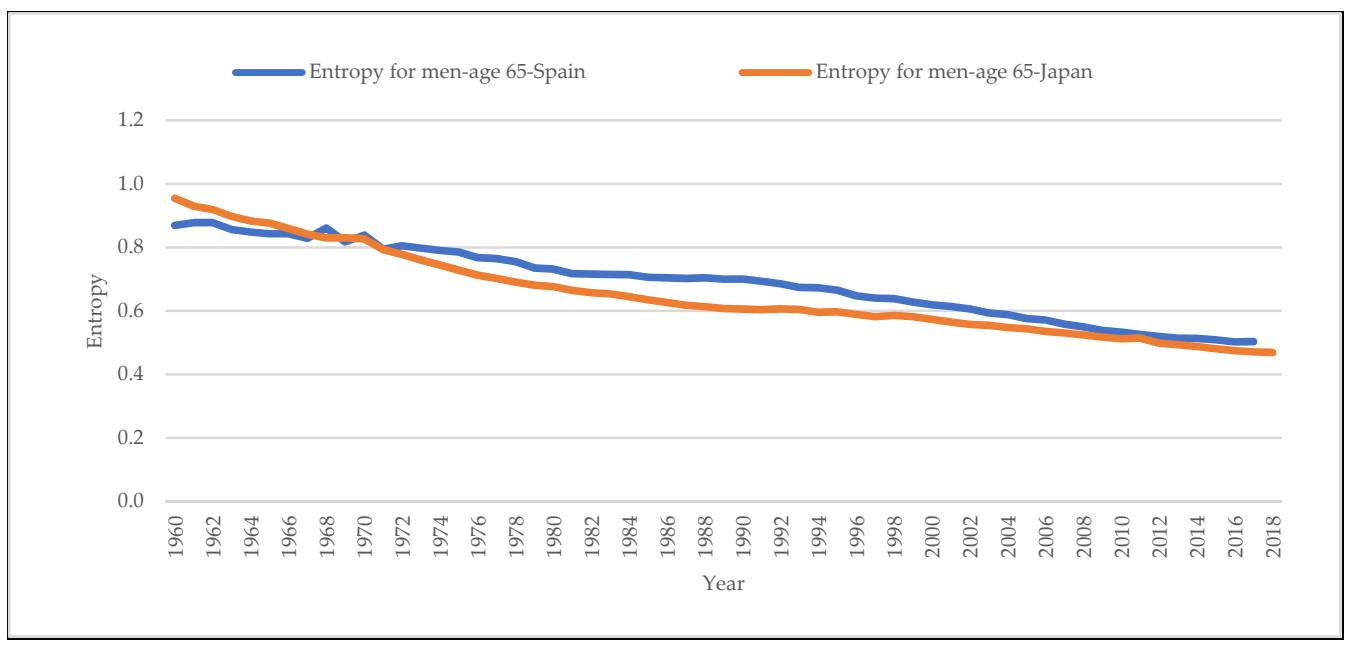

Figure 8. Entropy evolution for the male population at 65 over calendar years. Source: authors.

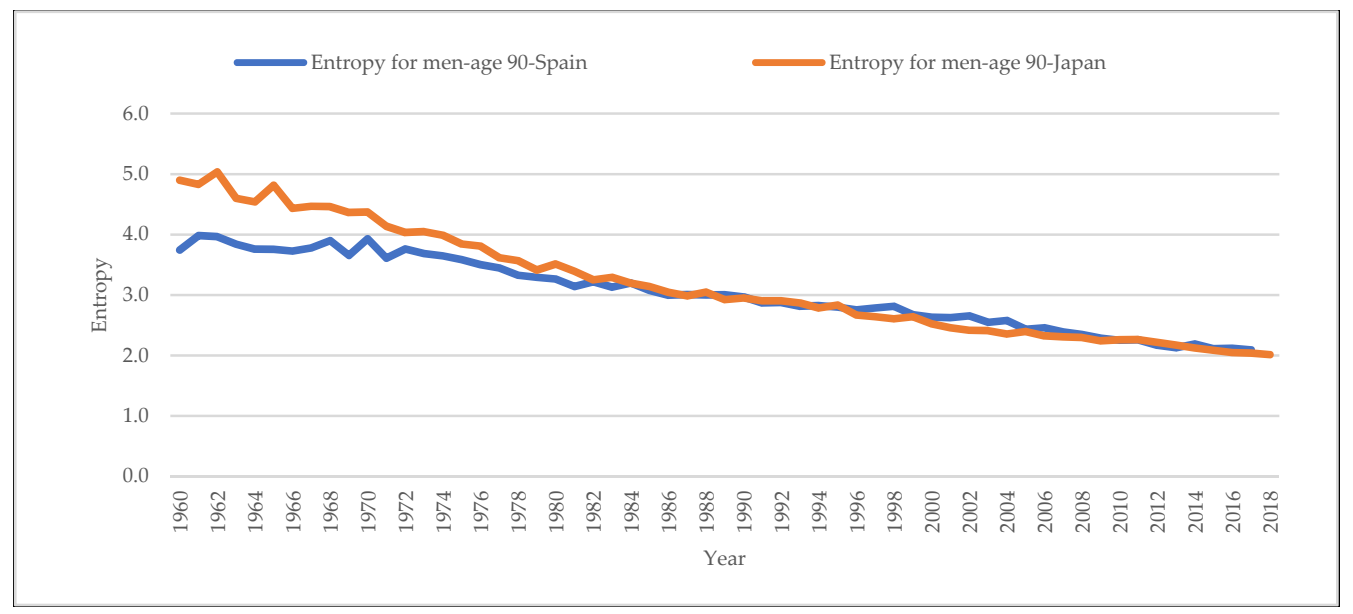

Figure 9. Entropy evolution for the male population at 90 over calendar years. Source: authors.

Measuring mortality using entropy, using the Keyfitz indicator [15], is quite different when we compare it by calendar years instead of by people's ages. The first thing that we note is that its progression is different for each of the ages selected. Furthermore, the evolution of the measurements differs for each population. It therefore seems obvious that the entropy index allows the differences in the improvements of mortality reflected in its acceleration or deceleration processes to be clearly perceived.

In the case of Japan, the process steadily decreased and at a quiet regular intensity, even though at a greater intensity in the initial calendar years with the intensity reducing toward the end of the last century, before increasing in intensity, although more slowly than in the first part of the selected period. However, the opposite occurs in the case of the Spanish population. The intensity fell over the initial years of the period and even managed to increase.

This shows that there was a clear difference in the behaviour in the improvement to mortality rates behaved clearly different in each of the countries. We checked it using the entropy index, which allows us to perceive a clear acceleration in the first third of the period and a deceleration in the second. We also find that the entropy is lower, practically for the years in the whole period in the case of Japan.

Something similar occurs for the 65-year-old, but with less intensity. Even in the case of Spain, there is a steady downward trend for the whole selected period, except in the central section. This means that the difference between the entropies is lower than what occurs at 40 . We also find an interesting behavior, and that is that the entropy in the case of 
Spanish men is lower than that for Japanese men. The acceleration or deceleration process is very clear in the Japanese population.

However, the entropy measurement clearly changes at 90 . The first thing found is that, even though the baseline gross data has been smoothed, the entropy measurement is jagged for those very old ages. Furthermore, the entropy in the case of Japan is much higher than the Spanish one until nearly halfway through the period. The size of the entropies of both populations swaps from then onwards. The entropy for the year 2000 drops with greater intensity. Therefore, the measurement we apply here is a suitable indicator of the acceleration of the improvement by the Japanese population in the first part of the period, before both populations then align again in the last years.

\subsection{Detailed Analysis of the Results Obtained for the Female Populations}

Focusing our study on the comparative analysis for the female case, the results obtained are those presented in the following Figures 10-12:

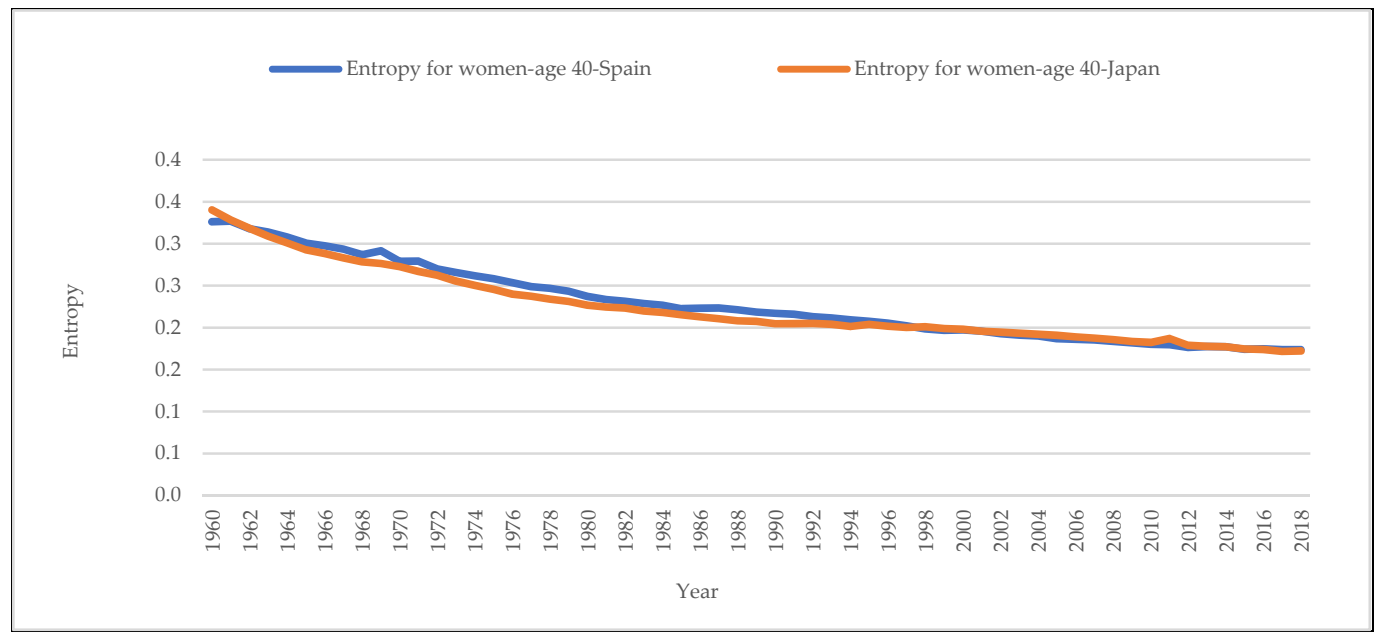

Figure 10. Entropy evolution of the female population at 40 over calendar years. Source: authors.

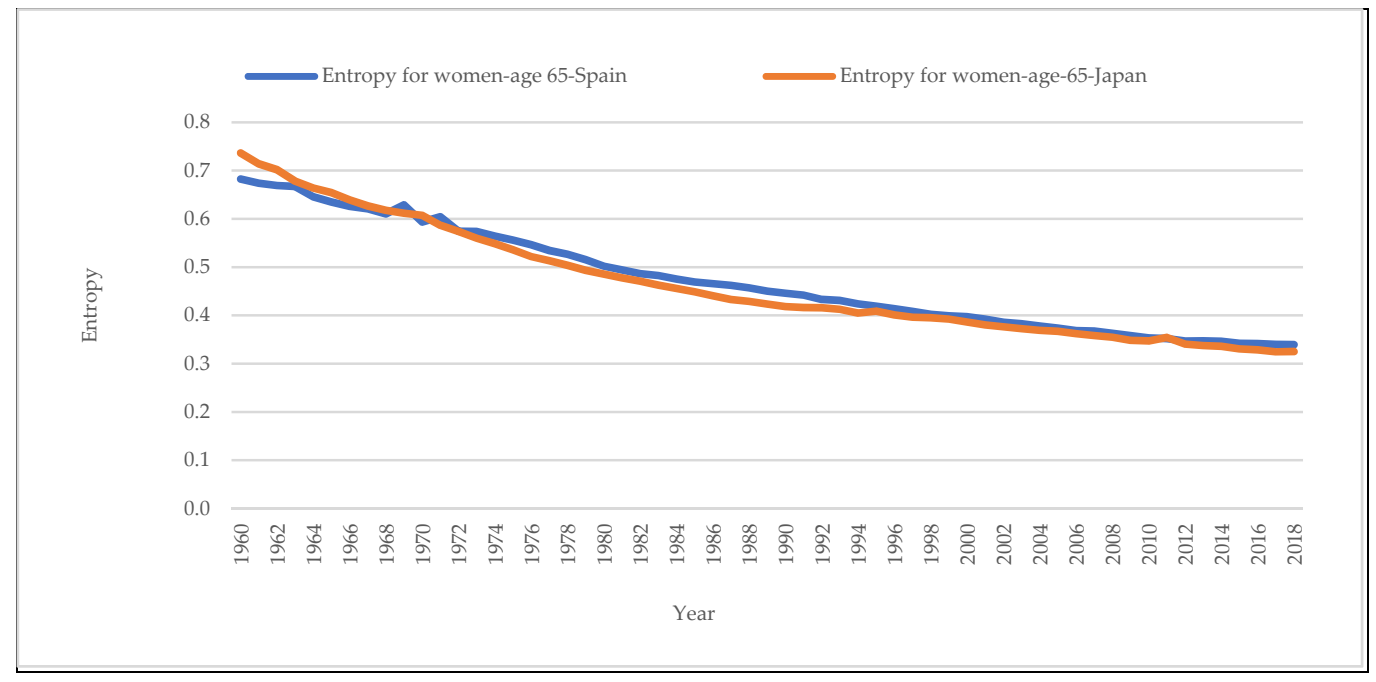

Figure 11. Entropy evolution of the female population at 65 over calendar years. Source: authors. 


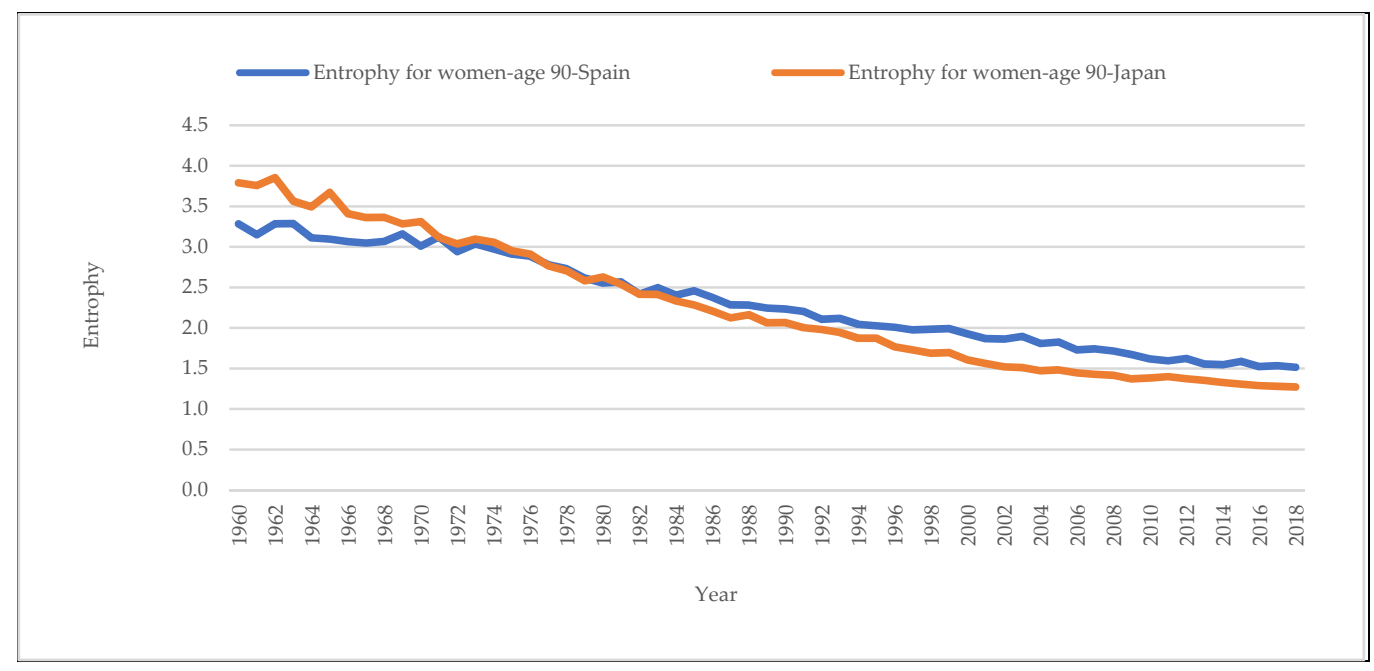

Figure 12. Entropy evolution of the female population at 90 over calendar years. Source: authors.

The first observation we should make is that the entropy measurement does not significantly differ for a Japanese female population aged 40 with respect to a Spanish female population of the same age. The downward intensity in the first part of the period is clear, slows down in the central sector, and gently accelerates in more recent years. Therefore, the entropy in both populations has evolved in a very similar way in terms of size and intensity, particularly in this century.

Even though the entropy in both populations at 65 also runs parallel, the acceleration toward improvement is greater. Above all, in the case of the Japanese population.

At the age of 90 , the jagged measurement already mentioned for the male population can also be clearly perceived in the case of women. As regards the size of the index and its evolution, we find that the evolution of the entropy is much higher in the Japanese population in the first 15 years of the period. They both then level out around 1975, and the entropy of the Japanese population is then lower than the Spanish one, with the gap remaining to the present, even though reducing very slowly. As regards its size, we find that its value is clearly higher, initially in the case of the Japanese population, to cross with the Spanish case in around 1975 and remain below until the present. The conclusion is therefore clear. The Japanese population showed greater improvement during the whole period considered.

The following Tables 4 and 5 are the summary of the results from Sections 5.1 and 5.2 and that were displayed graphically for clearer understanding.

As regards the age of 40 , the indicator shows that Japan begins with a magnitude higher than the one for Spain, as the result of higher mortality. It drops over time and stays under the values for Spain, indicating a greater acceleration of mortality. The Spanish population shows a clear deceleration between 1980 and 1990.

The indicator for the age of 65 shows that the mortality of the Japanese population is clearly higher, at the start of the period, but from 1970 onwards, it falls below the figure for Spain and remains so to the present. That means that the drop in mortality in the population of Japan accelerated faster than that of Spain until roughly 1980. From then onwards, it decelerates to close to Spain's evolution of the improved mortality.

The indicator for age 90 shows that Japanese mortality is clearly higher than the rate for Spain in the first years of the study period. After 1980, the indexes cross and remain at a similar level until roughly 1995 . During that time period, mortality continues to improve for both populations. From that date onwards, both improvements evolve, almost in parallel, in general, even though the entropy index for Japan remains under the Spanish one. 
Table 4. Entropy values for the male population for Japan and Spain, for three specific ages: 40, 65, and 90 years old, and for different calendar dates.

\begin{tabular}{|c|c|c|c|c|c|c|c|}
\hline \multicolumn{8}{|c|}{ Men (Age 40) } \\
\hline & 1960 & 1970 & 1980 & 1990 & 2000 & 2010 & 2018 \\
\hline Japan & 0.405 & 0.343 & 0.295 & 0.272 & 0.265 & 0.244 & 0.226 \\
\hline Spain & 0.398 & 0.363 & 0.325 & 0.322 & 0.291 & 0.254 & 0.238 \\
\hline \multicolumn{8}{|c|}{ Men (Age 65) } \\
\hline & 1960 & 1970 & 1980 & 1990 & 2000 & 2010 & 2018 \\
\hline Japan & 0.955 & 0.826 & 0.676 & 0.605 & 0.573 & 0.512 & 0.469 \\
\hline Spain & 0.881 & 0.817 & 0.734 & 0.700 & 0.628 & 0.539 & 0.503 \\
\hline \multicolumn{8}{|c|}{ Men (Age 90) } \\
\hline & 1960 & 1970 & 1980 & 1990 & 2000 & 2010 & 2018 \\
\hline Japan & 4.898 & 4.370 & 3.513 & 2.952 & 2.522 & 2.257 & 2.012 \\
\hline Spain & 3.968 & 3.655 & 3.293 & 3.001 & 2.676 & 2.286 & 2.094 \\
\hline
\end{tabular}

Source: authors' calculation.

Table 5. Entropy values for the female population for Japan and Spain, for three specific ages: 40, 65, and 90 years old, and for different calendar dates.

\begin{tabular}{|c|c|c|c|c|c|c|c|}
\hline \multicolumn{8}{|c|}{ Women (Age 40) } \\
\hline & 1960 & 1970 & 1980 & 1990 & 2000 & 2010 & 2018 \\
\hline Japan & 0.340 & 0.273 & 0.227 & 0.205 & 0.198 & 0.182 & 0.172 \\
\hline Spain & 0.326 & 0.279 & 0.237 & 0.217 & 0.197 & 0.180 & 0.174 \\
\hline \multicolumn{8}{|c|}{ Women (Age 65) } \\
\hline & 1960 & 1970 & 1980 & 1990 & 2000 & 2010 & 2018 \\
\hline Japan & 0.736 & 0.607 & 0.486 & 0.418 & 0.386 & 0.347 & 0.325 \\
\hline Spain & 0.682 & 0.594 & 0.502 & 0.446 & 0.397 & 0.353 & 0.339 \\
\hline \multicolumn{8}{|c|}{ Women (Age 90) } \\
\hline & 1960 & 1970 & 1980 & 1990 & 2000 & 2010 & 2018 \\
\hline Japan & 3.789 & 3.311 & 2.628 & 2.063 & 1.605 & 1.381 & 1.271 \\
\hline Spain & 3.283 & 3.007 & 2.552 & 2.230 & 1.926 & 1.616 & 1.514 \\
\hline
\end{tabular}

Source: authors' calculation.

We can conclude by pointing out that it is not possible to speak of the sustainability of the longevity acceleration for any of the male populations studied.

For age 40, the indicator shows that the mortality for the population of Japan is initially slightly higher than that of Spain but drops after a few years, without the difference being significant. This indicates that there is greater acceleration in the improvement to mortality for the Japanese collective. From roughly 1995 onwards, the difference between the two populations is not significant.

For age 65, the indicator shows that the mortality of the Japanese population is higher. From 1970, it drops under the Spanish indicator and remains steady to the present. That means that the drop in mortality in the population of Japan accelerated faster than that of Spain until roughly 2005. In the following years, there is a greater deceleration in the Japanese population, without that even coming near in any case.

The indicator for age 90 shows that the mortality of the Japanese population is clearly higher than the Spanish in the first years of the study period. Shortly after 1970, the indexes cross and remain at a similar level until roughly 1982. This indicates that the acceleration of the improvement in Japan is clearly greater than that of Spain. From then onwards, i.e., 1982, improvement accelerates in the case of the Japanese population until 2005, approximately, which is when Japan's improvement decelerates. 
Given everything relating to the last table, sustainability of the longevity acceleration can neither be claimed for the female population.

According to this indicator, everything seems to point to that the deceleration was greater in the case of women than of men in the last two decades. It also shows that the index is greater at older ages, which is consistent with greater mortality. In general, it also clearly shows a smaller magnitude for more recent dates. This behavior is consistent with lower mortality and is even inverse to the mathematical life expectancy, as justified in the Formula (4).

\section{Discussion, Managerial Implications, Limitations, and Future Lines of Research}

The present article is relevant because it shows another type of indicator of the improvement of mortality rate and, therefore, of the aging of a country. The entropy indicator is different from the classical one, which is based on the life expectancy to "measure" the progress of the aging of a country's population.

The previous research mostly focused on absolute measures, such as the increase in the number of years of life, while our research provides results relating to the intensity of the improvement in mortality, not only in the number of years of progress but also in the intensity in which it is progressing and whether this intensity is increasing or decreasing, i.e., whether the improvement in mortality is accelerating or decelerating. This, in turn, makes it possible to compare the evolution between two different countries. Having stressed the importance of our contribution, we believe that this paper fills a gap in the existing literature.

An important advantage that we find when applying the entropy indicator is that it allows us to measure the effects of a change in the mortality rates, reducing it to an index that is consistent with a single figure.

Given the results obtained, we believe that the entropy measurement is a suitable tool to analyse improvement to mortality and determine where longevity accelerates or decelerates.

On the one hand, we find interesting evidence when studying the behavior of entropy during the selected calendar period, as can be seen in Figures 7-12. When the trend is downwards, it represents an improvement in mortality and, therefore, survival increases, and vice versa. Furthermore, the intensity of the trend shows whether it is the product of acceleration or deceleration.

On the other hand, we observe significant differences between countries and sex using the entropy indicator. We find that the acceleration of improvement in the male population (Figures 7-9) is greater in the Japanese case, above all in the initial years of the period than in the Spanish population. In the Spanish case, the improvement is more regular with an entropy higher than the Japanese one, practically throughout the period. The exception is the 90-year-old population and for the first 20 years. Regarding the female population (Figures 10-12), we find that the evolution is similar in both populations, even though there is mainly a slightly inferior entropy in the case of Japan.

In turn, taking into account the results obtained during the study, and bearing in mind the trigger question included in the title of our paper, we conclude that it cannot be claimed that the acceleration of longevity is sustainable, at least in recent decades, for such representative populations as Japan and Spain. This implies that the acceleration of longevity has not been maintained more recently.

All the conclusions previously mentioned are of particular interest as they provide information on the evolution of the improvement to the mortality rate, its acceleration or deceleration, in age ranges in which the insurance industry and financial institutions market their products. For ages under 65, the marketing is mainly focused on life insurance or mortgages. In the case of ages around 65 , it is focused on products related to retirement income or pension plans, and for older ages, the products are mainly reverse mortgages, dependency, and, in general, long-term care insurance. 
We therefore consider that this paper provides the managers of those entities with a valuable tool when making management present and future decisions. The entropy index makes it possible to find out whether the mortality rate is increasing at a higher rate than that foreseen in the life tables commonly used by insurance companies, pension funds, or financial institutions. It therefore enables these companies to adjust their life tables, and consequently, adjust their mathematical provisions in accordance with the risk obligations contracted. We believe that this is the correct way to maintain their mandatory mathematical provisions at all times, in the amount corresponding to the obligations contracted with their clients, ensuring them the guaranteed annuities toward the future.

From a business managerial point of view, the innovative results obtained in this research imply a "wake-up call" to the heads of pension fund managers, insurance and financial companies, and pension and welfare institutions about the lack of foresight they have shown when designing and offering private pension plans, retirement plans, or life insurance policies, among others, in the face of progress in mortality improvement.

Regarding the limitation of the article, we note that the research results were limited for several aspects:

As we described above, we research the entropy indicator taking into account only the mortality improvement. That is, considering the overall variation of the mortality rate as a whole, including all the effects of the different factors influencing the mortality rate at the same time. A disaggregated analysis of the causes of death would include the incidence of each of them, such as cancer, cardiovascular disease, diabetes, Alzheimer's, or pneumonia, among others. The inclusion of all these factors has several advantages, but it also has some disadvantages. The main advantage is that it would improve the accuracy of the results, but on the other hand, achieving this would imply a high monetary and time cost due to the great difficulty and complexity of processing the data and the corresponding calculations. However, it represents one of our future research directions.

The inclusion of other important factors that influence the economy of a country in the entropy index, such as the economy itself, different social aspects, health, etc., would enrich the meaning and the representation of the results obtained. However, from our humble point of view, there would possibly be specific indicators in the existing literature regarding the economic field more appropriate to use than the entropy index, which we used from a demographic point of view. Nevertheless, we believe that it may represent another interesting research line toward the future.

Another limitation may be not to have included alternative countries in the present research. An example of those could be the case of Sweden, a country with a wide experience and breadth of information about mortality. Similarly, it would be interesting to research the mortality in the United Kingdom for its deep and historical studies in the field, or about the mortality in the United States of America because they represent a very different population scenario in terms of lifestyle, habits, or economic evolution, among others, which could provide interesting information about the evolution of mortality improvement creating a new additional research direction.

Finally, we propose the use of this tool when updating mortality tables, for example, for completed each calendar decade. This way of proceeding would avoid the lag in the case of those tables, as was the case decades ago, with pension funds, many of which went bankrupt due to failing to foresee the advance in longevity in due time. The updating at the end of each calendar decade of this indicator is part of the next study in our research line development.

Author Contributions: Conceptualization, A.B.Z. and A.J.B.Á.; data curation, A.B.Z. and A.J.B.Á.; formal analysis, A.B.Z. and A.J.B.Á.; investigation, A.B.Z. and A.J.B.Á.; methodology, A.B.Z. and A.J.B.Á.; resources, A.B.Z. and A.J.B.Á.; supervision, A.B.Z. and A.J.B.Á.; validation, A.B.Z. and A.J.B.Á.; visualization, A.B.Z. and A.J.B.Á.; writing-original draft, A.B.Z. and A.J.B.Á.; writingreview \& editing, A.B.Z. and A.J.B.Á. Both authors have read and agreed to the published version of the manuscript. 
Funding: This research received no external funding.

Institutional Review Board Statement: Ethical review and approval were waived for this study, due to the fact that, the database used in this paper is published on the Human Mortality Database website for academic or research purposes under free registration.

Informed Consent Statement: Not applicable.

Data Availability Statement: The database used in this paper is published on the Human Mortality Database website for academic or research purposes under free registration. Available online at: www.mortality.org, accessed on 26 May 2021.

Acknowledgments: We would like to thank Deborah Powel for her help with the English translation and editing.

Conflicts of Interest: The authors declare no conflict of interest.

\section{References}

1. Pollard, J.H. The Expectation of Life and Its Relationship to Mortality. J. Inst. Actuar. 1992, 109, 265-276. [CrossRef]

2. Vaupel, J.W. How change in age-specific mortality affects life expectancy. Popul. Stud. 1986, 40, 147-157. [CrossRef] [PubMed]

3. Wilmoth, J.R. Is the Pace of Japanese Mortality Declive Converging Toward International Trend? Popul. Dev. Rev. 2002, 24, 593-600. [CrossRef]

4. Nusselder, M.A.; Mackenbach, J.P. Rectangularization of the survival curve in the Netherlands, 1950-1992. Gerontologist 1996, 36, 773-782. [CrossRef] [PubMed]

5. Keilman, N.; Pham, D.Q.; Hetland, A. Why Population Forecast should be Probabilist-Ilustrated by the Case if Norway. Demogr. Res. 2002, 6, 431. [CrossRef]

6. Betzuen, A. Un análisis sobre las probabilidades de predicción de la mortalidad futura aplicando el modelo de Lee-Carter. An. Inst. Actuar. Españoles 2010, 16, 111-140.

7. Betzuen, A.J.; Betzuen, A. The possibility of a Longevity Index in the European Union under Solvency II guidelines. In Proceedings of the International Conference: IME 2015, 19th International Congress on Insurance: Mathematics and Economics, Liverpool, UK, 24-26 June 2015.

8. Betzuen, A.J.; Betzuen, A. La posibilidad de un índice de Longevidad para la unión europea bajo las directrices de Solvencia II. An. Inst. Actuar. Españoles 2016, 21, 73-106.

9. Betzuen, A.J.; Betzuen, A. Is there parallelism between the improvement in the survival of the Spanish and Japanese population? In Proceedings of the XXVIII AEDEM International Meeting, Tokyo, Japan, 3 September 2019.

10. Lee, R.; Carter, L. Modeling and Forecasting U.S. Mortality. J. Am. Stat. Assoc. 1992, 87, 659-671. [CrossRef]

11. Koissi, M.; Shapiro, A.; Hognas, G. Evaluating and extending the Lee-Carter model for mortality forecasting confidence interval. Insur. Math. Econ. 2006, 38, 1-20. [CrossRef]

12. Pitacco, E.; Denuit, M.; Haberman, S.; Olivieri, A.M. Modelling Longevity Dynamics for Pensions and Annuity Business. Insur. Math. Econ. 2014, 54, 12-27.

13. Renshaw, A.; Haberman, S. On age-period cohort parametric mortality rate projections. Insur. Math. Econ. 2009, 40, 255-270.

14. Tabeau, E.; Ekamper, P.; Huisman, C.; Bosch, A. Predicting mortality from period, cohort, or cause-specific trends: A study of four European countries. In Forecasting Mortality in Developed Countries: Insights from A Statistical, Demographic and Epidemiological Perspective; Tabeau, E., Van Den Berg Jeths, A., Heathcote, C., Eds.; Kluwer Academisc Publishers: Dordrecht, The Netherlands, 2001; pp. 159-187.

15. Keyfitz, N. Applied Mathematical Demography, 2th ed.; Springer: New York, NY, USA, 1985.

16. Shannon, C. A Mathematical Theory of Communication. Bell Syst. Tech. J. 1948, 27, 13-17. [CrossRef]

17. Khalaf-Allah, M. Stochastic Analysis of Longevity and Investment Risk in The Context of Life Annuities. Ph.D. Thesis, Degree of Doctor of Philosophy, Faculty of Actuarial Sciences and Insurance, City University, London, UK, 2007.

18. Noyer, A.; Coleman, C.M. A Universal Pattern of the Evolution of Life Table Entropy and Life Expectancy. In Proceedings of the PPA Annual Meeting, Boston, MA, USA, 1-3 May 2014.

19. Goldman, N.; Lord, G. A New Look at Entropy and the Life Table. Demography 1986, 23, 275-282. [CrossRef] [PubMed]

20. Hill, G. The entropy of the survival curve: An alternative measure. Can. Stud. Popul. 1993, 20, 43-57. [CrossRef]

21. Human Mortality Database. Available online: www.mortality.org (accessed on 7 April 2021).

22. Coughlan, G.; Epstein, D.; Ong, A.; Sinha, A.; Hevia-Portocarrero, J.; Gingrich, E.; Khalaf-Allah, M.; Joseph, P. LifeMetrics: A Toolkit for Measuring and Managing Longevity and Mortality Risk; Technical Document; JPMorgan Pension Advisory Group: London, UK, 2007.

23. Millossovich, P.; Villegas, A.M.; Kaishev, V. StMoMo: An R Package for Stochastic Mortality Modelling. In Proceedings of the 7th Australasian Actuarial Education and Research Symposium, Gold Coast, QLD, Australia, 7-8 December 2015.

24. Aburto, J.M.; Alvarez, J.A.; Villavicencio, F.; Vaupel, J.W. The threshold age of the lifetable entropy. Demogr. Res. 2019, 41, 83-97. [CrossRef] 
25. Haberman, S.; Khalaf-Allah, M.A.E.; Verral, R.J. Entropy longevity and the cost of annuities. Insur. Math. Econ. 2011, 48, 197-204. [CrossRef]

26. Nagnur, D. Rectangularization of the Survival Curve and Entropy: The Canadian Experience, 1921-1981. Can. Stud. Popul. 1986, 13, 90-95. [CrossRef] 